\title{
Correction to: Artificial Intelligence and Machine Learning in Healthcare
}

\author{
Ankur Saxena and Shivani Chandra
}

\section{Correction to: \\ Ankur Saxena and Shivani Chandra (eds.), Artificial Intelligence and Machine Learning in Healthcare, https://doi.org/10.1007/978-981-16-0811-7}

Owing to an oversight on the part of Springer, this book was published as an authored book and the chapter author details were inadvertently removed during the production process. However, this book has now been updated with this erratum and chapter authors' details have been included in the table of contents and each chapter's opening pages. 